The effects of adult guidance and peer discussion on the development of children's representations: evidence from the training of pedestrian skills

Andrew Tolmie, James A. Thomson, Hugh C. Foot, Kirstie Whelan, Sheila Morrison \& Brian McLaren

Department of Psychology, University of Strathclyde

[The research reported in this paper was funded by the UK Department for Transport (Project No. S214H) and the Economic and Social Research Council (Grant No.

R000222468). The support of both is gratefully acknowledged.] 


\title{
The effects of adult guidance and peer discussion on the development of children's representations: evidence from the training of pedestrian skills
}

\begin{abstract}
It was hypothesised that practical training is effective in improving children's pedestrian skills because adult scaffolding and peer discussion during training specifically promote E3 level representation (linguistically-encoded, experientiallygrounded, generalisable knowledge), as defined by Karmiloff-Smith's (1992) representational redescription (RR) model. Two studies were conducted to examine in detail the impact of this social input, in the context of simulation-based training in roadside search skills. Five- to eight-year-olds were pre-tested on ability to detect relevant road crossing features. They then participated in four training sessions designed to promote attunement to these, under peer discussion condition vs adult guidance conditions (Study 1), and adult-child vs adult-group conditions (Study 2). Performance at post-test was compared to that of controls who underwent no training. Study 1 found that children in the adult guidance condition improved significantly more than those in the peer discussion or control conditions, and this improvement was directly attributable to appropriation of E3 level representations from adult dialogue. Study 2 found that progress was greater still when adult scaffolding was supplemented by peer discussion, with E3 level representation attributable to children's exploration of conflicting ideas. The implications of these findings for the RR model and for practical road safety education are discussed.
\end{abstract}




\section{Introduction}

Until recently, road safety education in Britain focused primarily on classroom-based teaching of general rules intended to specify action under many circumstances (e.g. the Green Cross Code's “stop, look, listen, think"), obviating any need for children to learn how to deal with individual situations (Thomson, Tolmie, Foot \& McLaren, 1996). In practice, however, children commonly fail to see how such rules relate to actual events (see e.g. Ampofo-Boateng \& Thomson, 1990), and knowledge of rules is commonly unrelated to road-crossing performance (Ryhammer \& Bergland, 1980).

For many branches of developmental and cognitive theory these difficulties are not surprising. As Thomson et al. (1996) note, there is a consensus that learning proceeds from the specific to the general, from action to representation. The start point is therefore typically participation in an activity, not generalised instruction. This position can be ascribed to both Piagetian and Vygotskian theory (Piaget, 1985; Vygotsky, 1978), given their constructivist nature; and to Gibsonian theory and connectionist modelling (Gibson, 1966; Bechtel \& Abrahamsen, 1991), which both characterise learning as the abstraction of invariances from specific inputs. Among contemporary approaches to developmental theory too, Karmiloff-Smith's (1992) representational redescription (RR) model proposes a general four-level sequence of developmental change, in which initially context-bound procedural knowledge (implicit or I level representations) is gradually transformed into explicit formulations (E level representations), making it available in other contexts, first to the self (E1 and E2 levels) and then to others via encoding in language (E3 level).

The implication is that in the ordinary course of events generalisable pedestrian skills are built up over time from specific encounters with traffic environments. To be successful, then, road safety education needs to provide experience of making actual road crossing decisions in safe and controlled contexts, affording the chance both to learn appropriate patterns of behaviour and to extend these to other situations. The efficacy of this approach has been confirmed in the Netherlands (Rothengatter, 1984) and in the UK. Thomson \& Whelan (1997) examined the impact of training sessions in which adult volunteers worked with triads of 5- to 6-year-olds on the identification of safe places to cross roads (i.e. start points and routes which avoid dangers such as reduced visibility and spending too long on the road). Children were taken to various 
locations, where they were set road crossing goals, and engaged in dialogue about the features that would make some routes unsafe, and how these might be dealt with (e.g. by moving where visibility was better). The effects of training were gauged from children's unsupported road crossing solutions at a pre-test, and on post-tests shortly after training and three months later. Children who had four to six half-hour sessions over a month showed robust improvements, making three times as many safe route decisions, and performing above the level typical of untrained 10-year-olds (Tolmie, Thomson, Foot, Whelan, Sarvary \& Morrison, 2002).

In related work, Ampofo-Boateng, Thomson, Grieve, Pitcairn, Lee \& Demetre (1993) trained 5-year-olds individually in safe place identification either at the roadside or on a table-top model with comparable road layouts and 'parked' toy cars. Both produced substantial improvements in selection of safe crossing places, with trained children able to articulate and explain solutions of which they showed no knowledge at pretest. Since all pre- and post-testing occurred at the roadside, these improvements must moreover have depended upon generalisation across contexts amongst those trained on the model. Thus practical training methods of the type employed by Thomson and colleagues apparently have the potential to promote not just improved performance, but explicit, transferable knowledge equating with E3 level representation (conscious knowledge available in other contexts and communicable to others) in the RR model.

If there is a gap in this work, it is that the mechanisms by which training produces high-level representation are unclear. No record of the content of training sessions was kept by either Ampofo-Boateng et al. (1993) or Thomson \& Whelan (1997). It is therefore difficult to determine whether improvements in children's performance depended on the exogenous influence of dialogue with trainers or (where relevant) other children; or, conversely, on endogenous processes following exposure to roadcrossing problems, as the RR model assumes (Karmiloff-Smith, 1992). There are signs that cognitive engagement with the problems was influential, since untrained children employed as controls in Thomson \& Whelan's (1997) research showed improvement pre- to post-test, despite the absence of feedback from testers. There are also cogent reasons, however, to suppose that it was trainer activity that was critical. 
Vygotsky (1978) proposed that children learn primarily by taking over control of actions initially performed under guidance: tutors use language to help direct the child's activity, and by appropriating and then internalising the linguistic encoding of action this involves, the child becomes able to direct him- or herself. Wood (1986) offers a similar formulation. Tutors employ physical demonstrations (e.g. putting two pieces of a puzzle together) to help children establish basic procedures (cf. I level representations). Once these procedures are in place, though, they switch to increasingly oblique verbal directions which embed procedural knowledge within explicit and generalisable strategies (e.g. "how about starting by putting the edge pieces together?"). Both accounts point to the same mechanism as producing E3 level representations in the earlier research: the encoding in language of problem elements and problem solutions by adult trainers. Support for the effects of linguistic encoding on shifts in representational level is provided by Pine, Messer \& Godfrey (1999). Children aged 5 to 7 years who saw demonstrations of solutions to balance beam problems and heard explanations of these progressed in understanding more than those who worked alone, and as much as those who were guided to their own solutions, suggesting that verbal encoding was the crucial influence on learning.

Interaction between the children being trained in groups in Thomson \& Whelan (1997) may also have contributed to learning. For complex problems where many solutions are possible, as in road-crossing, the application of knowledge to different contexts depends not just on it having been made explicit, but also on an ability to decide which elements of knowledge to accord greatest weight in any given instance. The assessment of different viewpoints that commonly arises in discussion between peers may therefore have provided children being trained with important experience of explicitly rehearsing this decision-making process, helping transform procedural knowledge into a more explanatory conceptual framework. Certainly, there are established effects on conceptual grasp of the evaluation of alternative ideas during peer collaboration (e.g. Howe, Tolmie, Greer \& Mackenzie, 1995; Tolmie, Howe, Mackenzie \& Greer, 1993; Williams \& Tolmie, 2000). More specifically, there is evidence that such effects extend to understanding of the rationale guiding procedural decisions (Howe, Tolmie, Duchak-Tanner \& Rattray, 2000; Miell \& MacDonald, 2000; Pine \& Messer, 1998; Tolmie, Howe, Duchak-Tanner \& Rattray, 1999). 
These arguments are necessarily speculative in the absence of more direct evidence. The way forward would be to make systematic comparison of the effects of different versions of the same training programme permitting a) only adult guidance, as in Ampofo-Boateng et al. (1993); b) only peer discussion, and c) a combination of the two, as in Thomson \& Whelan (1997). This would allow the unique and the additive or interactive effects of these potential influences to be gauged. Clear linkage between the acquisition of generalisable E3 level representations and one or other type of interaction during training would establish the influence of this as central to the outcome of practical pedestrian training, and demonstrate that individual cognition was not the key determinant of progress.

The present paper reports on two studies designed to make the proposed comparison. Study 1 contrasted the effects of adult guidance of individual children with those of peer discussion, whilst Study 2 compared the impact of adult guidance of individuals and groups. These studies had two principal aims. The first was to establish whether practical training results, as hypothesised, in the growth of explicit, communicable knowledge (i.e. E3 level representations) which assists generalisation of performance. The second was to examine whether this development, assuming it occurs, can be attributed to adult encoding in language of problem elements or peer discussion of alternative ideas. In order to provide the necessary standardisation and control of training sessions, both studies used computer simulations to train 5- to 8-year-olds in roadside search skills (i.e. direction of attention to features relevant to road-crossing, such as vehicle movements and road layout). This was chosen as the focus of training because Tolmie, Thomson, Foot, McLaren \& Whelan (1998) report that 6- to 8-yearolds perform poorly in this area, differentiating relevant from irrelevant features (e.g. vehicle colour, a man on a ladder against a house) at no better than chance level both on simulations and at the roadside. Children in the present research were therefore likely to possess little explicit knowledge of what to attend to prior to training, permitting development to be more readily detected. Moreover, if the outcomes for roadside search skills were consistent with those observed for safe place location, this would establish the general nature of the processes involved.

\section{STUDY 1}


Study 1 assessed the independent impact of adult guidance and peer discussion on children's roadside search skills. Identical simulation-based training exercises were carried out either by individual children working under the guidance of an adult volunteer, or else by small groups of children working collaboratively on their own. These exercises required them to view a series of animated traffic scenarios, and to decide if and when it would be safe for a figure depicted at the roadside to cross. To solve these problems, children needed to learn to attend to road layout and vehicle movements, and ignore other features. Progress was assessed via pre- and post-tests both on computer and at the roadside.

Before commencing the research, it was necessary to identify the dialogue elements characteristic of adult guidance and peer discussion, so that the incidence of these could be reliably extracted from recordings of the training sessions. The process of contingent support (Wood, 1986) is central to generic accounts of adult guidance. This involves the adult intervening to steer the child only when they falter, and then at the least directive level that achieves the desired result (e.g. prompting rather than demonstrating), since this encourages autonomy and appropriation of control of previously supported action. Recent research (e.g. Howe \& Tolmie, 1998; Tolmie et $a l ., 1999)$ identifies four types of dialogue as characteristic under these conditions: 1) instructions; 2) suggested actions; 3) questions requesting ideas about what to do; and 4) prompts which draw attention to factors not yet taken into account. These elements primarily constitute different forms of guidance, deployed according to the progress exhibited by the child, with a shift from instruction towards prompting occurring as children's competence improves.

Peer discussion is typified by the process of socio-cognitive conflict (Piaget, 1932; Doise \& Mugny, 1984). Where individuals present incompatible interpretations of an event, this is argued to cause conceptual conflict. This in turn provokes a process of conflict reduction via individual or joint construction of new concepts that resolve the discrepancies by combining aspects of the alternative ideas available. Research (e.g. Howe et al., 1995; Tolmie et al., 1993; Tolmie \& Howe, 1993) pinpoints a sequence of three types of dialogue as characteristic of this process amongst younger children: 1) suggestion of an idea; 2) disagreement with that idea; and 3) explanation justifying the original idea. Related research (see Tolmie \& Howe, 1994) indicates that chairing 
dialogue (i.e. interactions specifying what should be done by whom) is characteristic of unproductive peer discussion, this being what children fall back on when they are struggling with the task in hand and lack the means to progress.

Using this specification of anticipated dialogue elements, the objectives of Study 1 were to examine: 1) whether the two training conditions led to different degrees of improved performance post-training; 2) whether either led to increased incidence of E3 level representations and associated generalisation of performance from computer to roadside; and 3) whether observed changes in performance could be related directly to the incidence of the identified dialogue types.

It was anticipated that if adult guidance had positive effects, these would be indicated by a shift over the course of training from instruction towards prompting, and concomitant growth amongst children of explicit knowledge used to direct attention both on simulations and at the roadside. Since this growth has been argued to derive from appropriation of adult encoding of procedures and strategies in language, it ought to be directly related to the extent of such input from adults (i.e. the number of adult turns). If peer discussion had positive effects, this should be evident from an association between growth of explicit knowledge and the number of explanations generated by children following disagreement over individual suggestions, since these explanations would provide the fuel for conceptual growth.

\section{Method}

\section{Design}

The study employed a pre-test-intervention-post-test design. Children were pre-tested on computer and at the roadside for attention to features relevant to road crossing decisions; and, at the roadside only, on actual crossing judgements. Approximately three-quarters of the sample then worked through four training sessions designed to promote attention to relevant features, either in adult-child dyads (adult guidance condition) or in threesomes (peer discussion condition). The remaining children formed a control condition and received no training. Once training was complete, all children were post-tested using the same methods as the pre-test. Pre- and post-test performance was assessed via measures of 1) pick-up of relevant features relative to irrelevant; 2) explanations of relevance; and 3) selection of appropriate crossing 
opportunities. Pre- to post-test change was analysed for differences across conditions, and for relationship to the incidence during training of dialogue of specified types.

\section{Participants}

50 children ( 32 boys and 18 girls) aged between 5 years, 8 months and 8 years, 6 months $($ mean 6,9$)$ took part in the study. These children were drawn from three classes in a socially mixed primary school in the West End of Glasgow. The vast majority (43) had English as the language of the home; class teachers confirmed the remainder were competent English speakers. The research was ethically approved and all children participated with the permission of their parents and Glasgow City Council Education Department.

\section{Materials and Procedure}

Pre-test. Children were individually pre-tested on three tasks, in the order below. Responses on the first two tasks were recorded on audiotape, and on the third via a laptop computer. No feedback was provided on performance.

1. Computer report task. Twelve animated road scenarios, selected from a larger set developed by Tolmie et al. (1998), were shown in the same randomised order to each child on a computer located in a quiet room within school. These presented systematic combinations of three levels of relevant feature (dynamic - traffic movements on straight roads; static - junctions, blind hills, parked vehicles; static plus dynamic junctions etc. with moving traffic), with four levels of distractor i.e. additional irrelevant features beyond those implicitly present in the scene (none; auditory, e.g. the sound of breaking glass; visual, e.g. a man on a ladder against a house; visual plus auditory, e.g. a mother calling her children). Each sequence lasted about 10 seconds, and included the figure of a child standing at the roadside. After each, the child was asked what they had seen and heard "that the child on-screen would need to know about in order to cross the road safely". Full responses were encouraged by asking "Did you notice anything else they would need to know about to cross safely?". Children were not asked why identified features mattered, to avoid disrupting their reports, but such comments were accepted if offered spontaneously. 
2. Roadside report task. A few days later, each child was taken to a location by the school gates, on a section of busy straight road with a crossroads and traffic lights at one end and a blind bend at the other. Standing with a researcher in a fixed position at the roadside, they were asked to say what they could see and hear that they would need to know about in order to cross the road safely. Full responses were elicited as in the computer report task.

3. Roadside crossing decision task. When the roadside report task was complete, the child was taken to a nearby pedestrian crossing and timed as they led a researcher across the road and back. The mean of these times was entered on computer as a measure of actual crossing time. The child was then taken to a position where they had a clear view of traffic in both directions, and asked to shout each time they thought it would be safe to walk straight across the whole road. A researcher stood behind the child to ensure they did not step out, whilst a second recorded the child's responses and the movement of vehicles in either direction on computer, using software devised by Demetre, Lee, Grieve, Pitcairn, Ampofo-Boateng \& Thomson (1993). The length of the task was five trials or ten minutes, whichever was shorter.

Training. After pre-testing, children were randomly assigned to one of three conditions: adult guidance $(\mathrm{n}=18)$, peer discussion $(\mathrm{n}=18)$, or control $(\mathrm{n}=14)$. In the adult guidance condition, children worked one-to-one with the same adult for four sessions on a computer-based training task. The adult was one of six volunteer MSc psychology students ( 3 males, 3 females), none of whom had specialist developmental background, who were each allocated three pupils, and simply asked to help them solve the problems presented by the computer. Pupils in the peer discussion condition worked through the same sessions, but in threes, without adult assistance. Groups were mixed-gender and comprised individuals from the same class. Membership was constant throughout.

The training materials were 14 animated traffic scenarios, similar to those employed at pre-test. Two were used for practice trials, and the remainder for training itself. The training scenarios were designed in blocks of three, with one item presenting a straight road, another a blind hill, and a third a blind corner. All depicted a flow of traffic containing a gap that would allow sufficient time for a figure shown at the 
roadside to cross. After the first block, however, traffic moved at varying speeds, and the size of the safe gap gradually became smaller, making it more difficult to detect. In the third block continuous distractors (e.g. men working on a roof) were present in every item, and in the fourth block discrete distractors (e.g. a plane flying over) coincided with the gap. For one item in each block, the figure at the roadside was positioned where their view would be obscured, rendering it unsafe to cross irrespective of the gap. The practice items took the same form as the simplest training scenarios, one showing the figure in a safe location, the other in an unsafe one.

The task in both training conditions was to watch each scenario and identify if and when it was safe for the figure to cross. Each sequence could be repeated until a decision was arrived at, and the action could be frozen during discussion. Once a decision had been made, participants pressed either a green "GO" button at the appropriate point, or a red "NOT SAFE" button if they thought the location was unsafe. Task directions and feedback were provided both on screen, and through a voice-over. After correct responses, participants advanced to the next problem; after incorrect, they viewed the scenario again and made a fresh decision. Children in adult-child dyads were allowed to enter decisions themselves, and those in groups were encouraged to take turns at doing so.

Training sessions took place in a separate room, starting two weeks after pre-testing. Each group and adult-child dyad worked through one session per week, with a researcher present in the background. Session 1 began with the two practice trials, which the researcher used to explain the task and software, and then moved on to the first training item. Sessions 2 and 3 started at item 5 and 9 respectively to ensure children were exposed to all levels of difficulty. Session 4 started again with item 1 to allow recapitulation. Each session was stopped after 25 minutes, except the first, which was allowed 30 minutes because of the practice items. All sessions were openly videotaped using radio microphones to record the audio channel.

Post-tests. A week to ten days after training finished, all children were post-tested using the same methods as the pre-tests.

\section{Scoring}


Pre- and Post-tests. Transcripts of each child's responses on the computer and roadside report tasks were used to derive four indices of performance on each at preand post-test. Reported features (defined as single attributes; e.g. "car" would be one feature, "green car" two) were coded as either relevant or irrelevant, depending on whether they could have any bearing on making a safe crossing ("car" being relevant, for instance, but "green" irrelevant). A count was then made of the number of relevant (R) and irrelevant (I) features identified across trials. These values were used to calculate a standardised measure of pick-up of relevant features relative to irrelevant, the ratio score, defined as $(\mathrm{R}-\mathrm{I}) /(\mathrm{R}+\mathrm{I})$. Positive values indicated that relevant features were in the majority. The fourth index was the number of spontaneous justifications given for relevant features that demonstrated explicit understanding (e.g. "the hill is dangerous because he can't see cars coming over it"; "you have to think about all the places that cars could come from"). Whilst such comments were relatively infrequent, they were taken as an unbiased sign of the application of generalised, communicable principles to the task in hand i.e. of E3 level representation. The reliability of coding of the primary elements of the report tasks was determined via independent scoring of 17 randomly selected transcripts (roughly one-twelfth of the total) by two researchers. Agreement for number of relevant features was $91 \%$, for number of irrelevant $98 \%$, and for number of justifications $93 \%$.

The software used to record data for the roadside crossing decision task automatically calculated for each trial: a) the size, in seconds, of the gap accepted as safe; b) the delay, in seconds, between the leading car of the chosen gap passing and the child responding (smaller values indicating greater ability to anticipate gaps); c) whether the chosen gap was a 'tight fit' (i.e. less than 1.5 times the child's crossing time); d) the number of missed opportunities (i.e. unselected gaps greater than 1.5 times the crossing time). These were used to calculate individual pre- and post-test values for mean accepted gap size, mean starting delay, the proportion of accepted gaps which were tight fits, and the proportion of missed opportunities out of the total available.

Training sessions. The videotapes of the training sessions were inspected to determine the incidence of the seven types of dialogue associated with adult guidance and peer discussion (taking suggestions as common to both), plus agreements (included as a check on consensus for decisions). For each dyad or group, a count was made of the 
number of statements in each session that fell into these eight categories. A count was also made of the number of problems for which correct solutions were identified, as a measure of on-task success. The full list of variables is given below:

1) suggestions ("it's safe to go now", "it won't be safe there")

2) agreements ("yes, I think so too")

3) disagreements ("no, not then", "I don't think that's right")

4) explanations ("it's safe 'cos no cars are coming", "he can't see over the hill")

5) chairing ("it's my turn", "we press this button now")

6) instructions ("press the button now", "forget about the man on the ladder")

7) questions (“when should you go?”, “why then?”)

8) prompts i.e. statements or questions directing attention to something not yet considered ("would having the hill there make a difference to crossing?", "don't forget about cars coming round the bend")

9) correct solutions

Coding for the adult guidance condition distinguished between whether dialogue turns (i.e. any statement coded into one of the above categories) were made by the adult or the child, since this was necessary to detect appropriation by children. No distinction was made between speakers in the peer discussion condition, as past research (Tolmie \& Howe, 1993) indicates that in peer activity exposure to dialogue predicts outcome better than who says what. As a check on reliability, 10 randomly chosen sessions (approximately 12\% of the total) were scored independently by two researchers. Correlations across cases between their values for the frequencies of dialogue in each category were all highly significant, ranging from +.79 to +1.00 , with a mean of +.94 .

\section{Results}

Pre- to post-test change. Analysis focused first on establishing comparability between the conditions at pre-test, and then on whether there were differences between them in progress to post-test, or any generalisation from computer to roadside mediated by E3 level representation, as indexed by justifications. As Table 1 shows, there were slight variations in pre-test performance, but none were significant. Performance overall was characteristic of this age group (see Demetre et al., 1993; Tolmie et al., 1998), with little differentiation between relevant and irrelevant features either on computer or at the roadside; large accepted gap sizes and starting delays plus many missed opportunities, indicating hesitancy; and occasional poor decisions in the form of tight 
fits. There was no coordination across contexts via E3 level representation. Justifications were infrequent (though their absence at the roadside was probably attributable to the demands involved in reporting 'live'), and they were uncorrelated with any other index, save the reporting of relevant features on the computer task $(r=$ $+.35, \mathrm{df}=46, \mathrm{p}=.007$, one-tailed, controlling for verbal fluency, as estimated by total number of features reported).

Table 1 about here

Performance improved from pre- to post-test, but not uniformly across conditions, as a series of two-way mixed ANOVAs (pre- vs post-test x condition) revealed. For the computer report task, children in the adult guidance condition alone exhibited a genuine shift across problems towards reporting relevant features, with both an increase in $\mathrm{R}$, as in the other conditions (for pre- vs post-test, $\mathrm{F}(1,44)=25.38, \mathrm{p}<$ .001 ), and a decrease in I (for the interaction between time of testing and condition, $\mathrm{F}(2,44)=5.28, \mathrm{p}=.009$; adult guidance $<$ peer discussion but not controls at .025 level using the Bonferroni test). As a result, positive change in ratio score was restricted to this condition (for the interaction effect, $\mathrm{F}(2,44)=8.39, \mathrm{p}=.001$; adult guidance $>$ peer discussion and controls at .025 level, using Dunnett's $\mathrm{C}$ for unequal variances). The adult guidance children also showed the largest increase in justifications (for the interaction effect, $F(2,44)=3.28, \mathrm{p}=.047$ ), and this was the only condition where change was actually significant $(\mathrm{t}(16)=2.94, \mathrm{p}=.005$, onetailed). Moreover, justifications and ratio score were significantly correlated at posttest in this condition $(\mathrm{r}=+.50, \mathrm{df}=14, \mathrm{p}=.025$, one-tailed, controlling for total number of features reported), suggesting greater selectivity in feature identification was a systematic effect driven by growth in explicit representation.

At the roadside, only accepted gap size and starting delay showed significant change $(\mathrm{F}(1,44)=7.32, \mathrm{p}=.010$; and $\mathrm{F}(1,44)=4.42, \mathrm{p}=.041$, respectively), and in neither case did this interact with condition. However, the adult guidance children exhibited the largest reductions in hesitancy, as measured by these variables, and were the only ones to show both an increase in $\mathrm{R}$ and a decrease in I. Moreover, for these children alone, increases in justifications on the computer task were correlated with increases at the roadside in $\mathrm{R}(\mathrm{r}=+.50, \mathrm{n}=17, \mathrm{p}=.022$, one-tailed $)$, and post-test justifications 
on the computer task were correlated with ratio post-test scores at the roadside, even when verbal fluency was controlled for $(\mathrm{r}=+.44, \mathrm{df}=14, \mathrm{p}=.046$, one-tailed $)$. In addition, increases in $\mathrm{R}$ on the computer task were associated with reduced starting delays $(\mathrm{r}=-.52, \mathrm{n}=17, \mathrm{p}=.015$, one-tailed), which were in turn associated with reductions in accepted gap size $(\mathrm{r}=+.61, \mathrm{n}=17, \mathrm{p}=.005)$. Thus the increases in E3 level representation evident in the adult guidance condition apparently led either directly or indirectly to increased attention to relevant features both on the computer and at the roadside, which led to more rapid assessment of traffic gaps, and concomitant reductions in accepted gap size. This generalisation underpinned by explicit representations was not only absent at pre-test, but remained so amongst children in the peer discussion and control conditions.

Relationship between change and interaction. Having established the effectiveness of the adult guidance condition, it was pertinent to consider how far interaction between children and adults was responsible for the observed growth in explicit representation. Table 2 shows mean values for the dialogue and performance indices by condition and training session, which indicate that the intervention conditions differed markedly in pattern of interaction. Two-way mixed ANOVAs (condition x session) confirmed this, revealing significant effects of condition, overall or in combination with session effects, on eight of the nine measures. The peer discussion condition was plainly less effective, since it produced static levels of correct solutions, whereas children in the adult guidance condition improved over time. Peer discussion children made many suggestions initially, and showed productive levels of disagreement and explanation (see Howe et al., 1995). However, explanations declined after session 1, and were less frequent overall than in the adult guidance condition. Disagreements, though, did not drop significantly, and were higher overall, whilst agreements fell away sharply. After the first session, then, peer exchange shed more heat than light, and the bulk of the dialogue was chairing. The loss of explanations, coupled with this focus on procedure, suppressed performance: across the four sessions, the fewer the explanations and the more the chairing, the fewer correct solutions dyads or groups arrived at (for explanations, $\mathrm{r}=+.41, \mathrm{n}=21, \mathrm{p}=.031$; for chairing, $\mathrm{r}=-.62, \mathrm{n}=21, \mathrm{p}=.001$, both one-tailed). It also had negative consequences for learning, since number of explanations was positively associated with pre- to post-test change in computer ratio score $(\mathrm{r}=+.31, \mathrm{n}=30, \mathrm{p}=.046)$. 
Table 2 about here

Adult-child dyads did better on-task because for them explanations remained constant, and the amount of chairing was substantially less. The positive association between performance and explanations merits attention, since it suggests interaction had its effect in this condition via specifically conceptual input. Initially, adult-child dialogue had the characteristics of scaffolding, with more prompts and questions than in the peer discussion condition, as adults helped children work things out for themselves. However, these elements of dialogue fell off as the number of correct solutions increased, and explanations became more frequent in relative terms $(4.6 \%$ of coded turns on Session 1 vs 7.3\% on Session 4). Moreover, as Table 3(i) shows, this change in emphasis was accompanied by a marked shift towards explanations being provided by the children. Adults made 3.5 times as many explanations as children on the first session, whereas something close to parity was achieved by the fourth (for the main effect of adult vs child, $(\mathrm{F}(1,30)=9.39, \mathrm{p}=.005$; for the interaction between originator and session, $\mathrm{F}(3,30)=3.42, \mathrm{p}=.021)$.

Table 3 about here

In order to discern what led children to start providing explanations, the videotapes of the adult guidance sessions were re-inspected and the type of turn preceding both adult and child explanations was noted. Tables 3(ii) and (iii) show the percentage of explanations following the four most frequent types of preceding turn for adults and children respectively. As can be seen, at first adult explanations occurred in roughly equal measure after adult prompts, adult questions and child suggestions. Thereafter, however, they began to appear following child explanations, and by the third session they occurred more frequently after child than adult turns (for the interaction between dialogue type and session, multivariate $F(9,7)=3.92, p=.043$ ). Children's explanations always occurred mainly in reply to adult prompts and questions, but they increased in frequency over the sessions, as already noted. At first, then, adults gave prompts and asked questions to direct children's attention, but also provided an explanatory commentary on these, and on children's suggestions. Over time, the provision of explanations following adult prompts and questions (and to some extent 
children's own suggestions) was taken on by the children themselves, whilst the adults maintained a (presumably corrective) commentary on this. Thus there were strong signs of appropriation by children of adult explanations in particular, rather than their linguistic encoding more generally. That this was genuine appropriation is confirmed by the fact that it was children's own provision of explanations that predicted subsequent change in justifications $(\mathrm{r}=+.52, \mathrm{n}=16, \mathrm{p}=.019$, one-tailed), the central element in improved performance at post-test.

\section{Discussion}

Children in the adult guidance condition exhibited clear signs of the generalisation of performance hypothesised to have occurred post-training between table-top model and roadside in Ampofo-Boateng et al. (1993), with systematic progress across problems on the computer being significantly correlated with increased attention to relevant features in real traffic environments and improvements in road crossing judgements. This generalisation, and all other aspects of progress, was mediated, as anticipated, by the development of explicit understanding, as indexed by justifications. Contrary to expectation, such understanding was not completely absent at pre-test, but it was limited, and there was no sign that it acted to direct attention so systematically. Pre- to post-test change for the adult guidance children was therefore not just a matter of development of explicit understanding, but of its deployment in the service of a variety of judgements. This explicit understanding thus met all the criteria for E3 level representations within Karmiloff-Smith's (1992) RR model. It was encoded in language and communicable to others, it was deployed flexibly and spontaneously, and it had an influence across contexts. Despite this, there was strong evidence that rather than being the product of endogenous processes, it derived from social interaction of a specific type, being acquired via appropriation of explanations from adults during the course of adult-structured activity (cf. Vygotsky, 1978). The lack of progress amongst control and peer discussion children, in spite of extensive testing, and, in the latter case, participation in the same intervention activity, would seem in contrast to rule out any effect of simple exposure to problems, at least on this time scale.

These data confirm the critical nature of social input within training of this kind. What was less expected was that the most influential form would be explanatory dialogue 
between adult and child, rather than more basic linguistic encoding of actions. Such dialogue has not typically been associated with adult guidance (see e.g. Damon \& Phelps, 1989; Howe \& Tolmie, 1998), and yet if a similar process had been operating in the one-to-one training employed by Ampofo-Boateng et al. (1993), it would account for the observed growth there in ability to explain solutions. This suggests that it may not be a one-off effect.

\section{STUDY 2}

If adult-child dialogue leads directly to conceptual growth, there seems little need to hypothesise any similar role for peer discussion. Indeed, it has been argued (Tomasello, Kruger \& Ratner, 1993) that children under the age of 7 are too young to possess the intersubjective awareness necessary to collaborate effectively, at least without training (Mercer, Wegerif \& Dawes, 2003; Webb, 1989). However, there were promising signs in the initial interactions among the peer discussion children in Study 1, and their subsequent exchanges were inappropriately focused rather than uncoordinated. It is more plausible then that when the problems were easier, they had enough knowledge to interact effectively (children did exhibit some grasp at pre-test), but when these became harder, they had insufficient insight for discussion to help, and fell back on going through the motions of doing the task. With better understanding, then, the proposed role of peer discussion in promoting explicit representation through justification and evaluation of conflicting tacit ideas might be more evident.

This suggests that adult guidance and peer discussion might interact productively if adults focused on supporting children's procedural grasp via scaffolding, and let discussion between children themselves serve as the means of developing explicit representations. In fact, since young children typically do not question the views of adults (Piaget, 1932; Kruger, 1993; Howe et al., 2000), the appropriation observed in Study 1 must essentially be a unidirectional and thus limited process. Between peers, more open exchange of ideas is likely, leading to greater explication of the conceptual basis of judgements. It was hypothesised, then, that adult-supported peer discussion would not only promote explicit representation as a direct function of the number of explanatory turns generated by children, but that this might have a greater impact on children's progress than adult guidance alone. To examine this hypothesis, Study 2 repeated Study 1, using the same method and materials, except that an adult was 
assigned to work with each peer discussion group, after being briefed on how to guide children towards solutions, whilst encouraging them to discuss the basis of their judgements among themselves. Since Study 1 had established that generalisation to the roadside was promoted by E3 level representations, attention was restricted to the relative impact of adult-child and adult-group conditions on the acquisition of these, and no roadside testing was employed.

\section{Method}

\section{Participants}

63 children ( 39 boys and 24 girls) from a primary school in a socially mixed area of East Glasgow took part in the study, in all cases with parental and local education authority permission, and with ethical approval. Their ages ranged between 5 years, 9 months and 7 years, 8 months (mean 6,6), and all had English as the language of the home. Eight parents (all female) of children at the school (in three cases, parents of participating children) were also recruited with the assistance of the headteacher to act as trainers, as in Thomson \& Whelan (1997). None had formal qualifications or experience relating to children, excepts as parents.

\section{Design and procedure}

The study employed the same pre-test-intervention-post-test design as Study 1, and used the same software for testing and training. Before the intervention, parent volunteers took part in an induction session which provided hands-on experience of use of the training software, scaffolding techniques, and ways of promoting discussion between children. Participating children were pre-tested individually using the Study 1 computer report task. They were then randomly assigned to one of three conditions: adult-child $(\mathrm{n}=24)$, where training was one-to-one; adult-group $(\mathrm{n}=24)$, where children were trained in groups of three; or control $(n=15)$, where no training was received. Training sessions were scheduled as in Study 1 and had the same format. Each adult was allocated six children for training, three within the adult-child condition, and three within the adult-group condition, groups being mixed gender and drawn from the same school class. Trainer allocations and group membership was constant throughout, and no parent was responsible for training their own child. All children were post-tested a week to ten days after training, using the computer report task. The indices of learning, on-task activity and dialogue were all scored as in Study 
1. Checks on the reliability of dialogue coding, based on 16 sessions $(12.5 \%$ of the total, half in each experimental condition) yielded agreement values close to those obtained previously (mean correlation $=+.87$, range $=+.78$ to +.97 ).

\section{Results}

Pre- to post-test change. Pre-test values for R and I (see Table 4) were somewhat lower than in Study 1, but ratio scores were very similar. Pre-test justification scores were also smaller, but were still marginally correlated with $\mathrm{R}$ score alone $(\mathrm{r}=+.20$, $\mathrm{df}$ $=60, p=.062$, one-tailed, controlling for total number of features reported). Since the materials and procedure were identical to Study 1, the implication is that the Study 2 children were somewhat less articulate, but displayed the same lack of discrimination between relevant and irrelevant features, and of coordination of judgement via explicit understanding. No significant effects of condition were found on any variable.

Table 4 about here

Outcomes for the two intervention conditions following training were close to those found for the adult guidance condition in Study 1. Whilst two-way mixed ANOVAs revealed significant overall shifts in both $\mathrm{R}$ and $\mathrm{I}(\mathrm{F}(1,60)=7.81, \mathrm{p}=.007$, and $\mathrm{F}(1,60)=4.49, \mathrm{p}=.038$ respectively), change was restricted to the two training conditions. Both showed the same pattern of significant increase in R (for adult-child, $\mathrm{t}=2.33, \mathrm{p}=.014$; for adult-group, $\mathrm{t}=2.21, \mathrm{p}=.018)$ and decrease in $\mathrm{I}(\mathrm{t}=-2.30, \mathrm{p}=$ .015 , and $\mathrm{t}=-2.54, \mathrm{p}=.009$ respectively, all tests one-tailed with $\mathrm{df}=23$ ) observed previously. The control children showed no significant change on either index. In consequence, both the intervention conditions made substantial positive gains in ratio scores, whilst the control children remained static, giving rise to a main effect of prevs post-test $(\mathrm{F}(1,60)=16.87, \mathrm{p}<.001)$ and an interaction between change and condition $(\mathrm{F}(2,60)=3.80, \mathrm{p}=.028)$. The extent of change in the adult-child condition was near-identical to that found in Study 1, but that for the adult-group was more than a third as much again. In both cases, the gain was highly significant $(\mathrm{t}=3.47, \mathrm{p}=$ .001 , and $\mathrm{t}=4.11, \mathrm{p}<.001$, respectively, both one-tailed with $\mathrm{df}=23$ ), although high variance meant that only change in the adult-group condition was significantly different from the controls ( $\mathrm{p}=.026$ using the Bonferroni test). 
Overall change in justifications was also significant $(\mathrm{F}(1,60)=8.11, \mathrm{p}=.006)$, but gains were greatest in the adult-group condition $(\mathrm{t}=2.48, \mathrm{p}=.01)$, and close to conventional significance levels for the adult-child condition $(t=1.65, p=.056$, both one-tailed with $\mathrm{df}=23$ ). The gain observed in the control condition was nonsignificant. More importantly, as in Study 1, even these modest shifts in explicit justification were accompanied by evidence of underlying changes in conceptual organisation. Thus, for both adult-child and adult-group conditions, justifications were correlated with ratio score at post-test, controlling for total number of features reported $(\mathrm{r}=+.42, \mathrm{p}=.023$, for both conditions). Moreover, in the adult-group condition the actual extent of change in justification directly predicted amount of change in ratio score $(\mathrm{r}=+.37, \mathrm{p}=.041$; all correlations one-tailed with $\mathrm{df}=21)$. The data suggest then that growth in explicit representation again drove the shift in attention towards relevant features, and that, as hypothesised, the gains were greater when adult guidance was supplemented by peer discussion.

Relationship between change and interaction. In order to establish how the observed gains were produced, dialogue during training was examined for systematic variations between conditions and patterns of association with pre- to post-test change. For the adult-child condition, the profile of dialogue and performance (see Table 5) was similar to Study 1, in that adults exhibited the same use of scaffolding. Thus questions and prompts started high, but declined as children's performance improved (for adult questions and number of correct solutions, $r=-.43, p=.018$; for adult prompts and correct solutions, $\mathrm{r}=-.37, \mathrm{p}=.037$; both one-tailed with $\mathrm{n}=24$ ). Children gave explanations from the outset, however, and these were now negatively correlated with number of correct solutions $(\mathrm{r}=-.50, \mathrm{n}=24, \mathrm{p}=.012$, two-tailed). This suggests child explanations were provided in response to request when performance was poor, rather than spontaneously, following appropriation. Consistent with this, child explanations were directly related to adult questions $(\mathrm{r}=+.59, \mathrm{n}=24, \mathrm{p}=.002$, two-tailed). These were also the main precursor of child explanations in Study 1, but they occurred more frequently here, perhaps because the briefing to adults to encourage child provision of explanations in the adult-group condition had a spill-over effect into the adult-child condition. As a result, the subtler influences on progress observed in Study 1 were apparently drowned out. The only significant positive influence on correct solutions was the total number of codable adult turns $(\mathrm{r}=+.41, \mathrm{n}=24, \mathrm{p}=.024$, one-tailed), of 
which chairing, questions and prompts constituted the bulk, indicating that on-task performance was primarily dependent on how much effort adults put into scaffolding.

This said, progress in terms of learning was very similar to Study 1, and there were signs that related mechanisms were at work, albeit more covertly. Child explanations were negatively associated with change in ratio score $(\mathrm{r}=-.35, \mathrm{p}=.048)$, and uncorrelated with change in justifications, but ratio change was related to adult explanations $(\mathrm{r}=+.39, \mathrm{p}=.031)$, the source of representational gains in Study 1 . Similarly, justification and ratio change were both related to number of correct solutions on-task $(\mathrm{r}=+.37, \mathrm{p}=.038$, and $\mathrm{r}=+.39, \mathrm{p}=.028$ respectively; all correlations one-tailed with $n=24$ ), which, as noted, was correlated with overall adult input. The first point is consistent with operation of the appropriation processes observed in Study 1. The second perhaps indicates the influence of more basic linguistic encoding, the mechanism originally hypothesised to underpin the effects of adult guidance on explicit representation, since adult input predominantly consisted of verbal descriptions of task procedures (chairing), plus deictic use of language with regard to features of the traffic environment (questions and prompts).

Table 5 about here

Dialogue and performance in the adult-group condition bore similarities to that in the adult-child condition. Correct solutions increased across training sessions in the same way (rather than remaining static, as in the Study 1 peer discussion condition), and differences between the two conditions in adult input were minor, confirming that the prior briefing of the trainers produced the same behaviour irrespective of condition. Thus prompts, the most important scaffolding behaviour, had similar incidence across conditions, and whilst questions were more frequent in the adult-group condition, this is explicable in terms of the requirements of managing three children rather than one. Correspondences between the conditions - and with Study 1 - were much less evident for child input, though. Chairing was higher in the adult-group condition, but whereas this was the dominant strand of group dialogue in Study 1, it was much lower here. Disagreements, potentially a key marker for exploration of ideas, remained a feature of discussion, in contrast to the adult-child condition, but explanations, a further key index, were now also more common than in the adult-child condition, where they 
were less common within the peer discussion condition in Study 1. As in the adultchild condition, explanations were in part a function of adult questions and performance level (for adult questions, $r=+.79, p=.009$; for correct solutions, $r-.75$, $\mathrm{p}=.015$; both one-tailed with $\mathrm{n}=8$ ). As a result, they were again high from the outset. However, they were also directly related to child disagreements $(\mathrm{r}=+.66, \mathrm{n}=$ $8, p=.037$, one-tailed). Agreements were also much higher than in the adult-child condition, and strongly related to child explanations $(\mathrm{r}=+.86, \mathrm{n}=8, \mathrm{p}=.003$, onetailed), indicating that airing of ideas typically resulted in consensus.

The implication then is that child explanations in the adult-group condition arose at least in part from self-sustaining discussion of ideas between children following disagreement. In this respect the data are in line with the hypothesised effect of supporting peer discussion. Moreover, the learning that occurred appeared to stem, as expected, from a different process to that in the adult-child condition. As there, the only positive influence on task performance during training was total adult input (for adult turns and correct solutions, $\mathrm{r}=+.61, \mathrm{n}=8, \mathrm{p}=.056$, one-tailed), confirming the positive effect of adult support. In this case, though, ratio and justification change were unrelated to either correct solutions or adult explanations. Evidence directly relating peer discussion to growth in explicit representation was absent, however: contrary to prediction, change in justification was uncorrelated with explanations or any of the other indices of child discussion.

\section{Discussion}

Study 2 provided confirmation of many aspects of the data reported in Study 1. Social input remained strongly associated with learning, whilst the lack of progress among control children showed again that exposure to problems during testing was not enough to produce detectable change. Growth in E3 level representations, as indexed by justifications, was again central to learning, with these generating a focus on relevant features at post-test that was absent at pre-test. In addition, representational growth in the adult-child condition was once more a function of adult input, though the appropriation of explanations noted in Study 1 was less evident. Progress instead appeared to be driven more by the basic linguistic encoding provided by adults, perhaps because of the lower levels of articulation and understanding apparent at pretest. 
Growth in the adult-group condition, despite the similarity of adult input, appeared to rest on different processes. Adult scaffolding helped counteract the unproductive elements (excessive chairing, disagreement without explanation) that characterised peer dialogue in Study 1, and facilitated exploration of ideas between children. The result was learning gains that, as hypothesised, were greater than those achieved when adults worked with children one-to-one. This conjunction of enhanced learning and productive peer dialogue (see e.g. Howe et al., 1995) strongly suggests there was a link between the two, despite the absence of evidence for a direct relationship, since the outcome is otherwise unaccountable. This absence would be explained, moreover, if the observed learning was the product of the internal reflection and re-equilibration that Piaget (1932) describes as following exposure to conflicting ideas, evident in past research from delays in learning following group discussion (Howe, Tolmie \& Rodgers, 1992).

\section{General Discussion}

The research reported above addressed two main issues. The first was whether it could be conclusively demonstrated that practical training in pedestrian skills using methods of the type employed by Ampofo-Boateng et al. (1993) and Thomson \& Whelan (1997) leads to the development of E3 level representations which assist generalisation of performance amongst children aged 5 to 8 years. The second was whether this outcome, if it occurred, could be directly attributed to hypothetically important processes of learner dialogue with adult trainers and other children. With regard to the first issue, if E3 level representations are defined as conscious knowledge available in other contexts and communicable to others (cf. KarmiloffSmith, 1992), there is little ground for disputing that the interventions employed in Studies 1 and 2 resulted in their development. In both cases, children in at least one training condition exhibited verbalisable understanding at post-test that was associated with systematic direction of attention to relevant traffic features across various situations, and in Study 1, across computer and roadside assessments. It is also plain that understanding of this kind was absent at pre-test: justifications during initial testing were not associated with a focus on relevant features over irrelevant, and no coordination of performance across contexts was apparent. 
On the second issue, dialogue during training was consistently linked with growth of E3 level representations among children who underwent one-to-one training with an adult. The position regarding children in the adult-group condition in Study 2 is less clear-cut, but there were again signs that representational change was brought about by on-task dialogue between children. The progress exhibited by children in the adult guidance and adult-group conditions stands in contrast to the absence of change among those in the control conditions, regardless of the amount of exposure they received via testing (greater in Study 1 than in Study 2, due to the use of roadside testing); and among children in the Study 1 peer discussion condition, who received equivalent exposure to those in the adult guidance conditions but showed no progress. It seems safe to conclude that, at the very least, certain forms of social exchange promote E3 level representation, and do so faster than mere exposure.

This said, the mechanisms that led to learning were not entirely as anticipated, since the effects of linguistic encoding of actions and explanatory dialogue did not equate straightforwardly with conditions of adult guidance and peer discussion. Indeed, three processes of growth were evident, rather than the two originally hypothesised. Thus in Study 1, adult guidance children progressed via appropriation of adult explanations, whereas in the equivalent condition in Study 2, growth seemed to depend more on appropriation of basic verbal encoding, in line with initial expectations. Resolution of conflicting peer explanations, for which there was evidence in the Study 2 adult-group condition, constituted a third process, apparently based on internal reflection.

This last process is of particular interest, since it implies an endogenous mechanism of representational growth, as presumed by the RR model, even if the operation of this was socially instigated - something Karmiloff-Smith (1992) acknowledges may sometimes occur. Both exogenous and endogenous processes would therefore seem to be possible, in this context at least. At the same time, however, the benefits of peer discussion in the Study 2 adult-group condition arguably depended on preliminary scaffolding, with representational growth coming about, as far as can be discerned, via the discussion of explanations between children which was made possible by adults' verbal direction of task procedures, and which originated more directly in responses to adult questions. It therefore rested on the framework, organisational and linguistic, that the adults gave to the activity. This suggests that exogenous encoding 
in language had functional precedence, and indeed, this was exactly why it was hypothesised that adult support for peer discussion would be effective. This process can be seen as a local manifestation of a more general sequence posited by Tomasello (1999). He argues that representational redescription is linguistically mediated, and derives from a capacity to take an outsider's perspective on one's own behaviour that originates in dialogues between adults and children during the course of adult guidance of activity.

If exogenous linguistic encoding is accepted as central to representational growth in both adult-child and adult-group conditions, it still remains necessary to consider more precisely how it operated from the perspective of the RR framework. In the context of roadside search skills, I level representations (implicit, context-bound procedures) would be expected to generate appropriate behaviours in some instances, but without any degree of control or flexibility, or coherence across problems. This would appear to be consistent with the lack of differentiation between relevant and irrelevant features exhibited by children at pre-test. However, the pre-test correlations between justifications and reporting of relevant features (but not ratio score) indicate that even at pre-test attention to relevant features was more conscious than this, though still unsystematic. Pre-test performance therefore more probably reflected E1 level representations, defined by Karmiloff-Smith (1992) as reduced descriptions or verbal tags that facilitate basic connections between events; or E2 level, defined as consciously accessible representations that allow fuller recognition of connections. The association of pre-test performance with verbalisation appears problematic for assigning it to E1 or E2 level, but Pine \& Messer (1999) argue on the basis of balance beam data that encoding in language is not in fact an exclusive marker of E3 level representations. In the present context, it is the correlation between justifications and ratio score that indicates pick up of relevant features which is both conscious and controlled, and this systematicity would seem to denote E3 level representation more than linguistic encoding as such. This suggests adult input initially acted to extend E1 representations to E2, by pointing up connections between problem scenarios. It then either shifted to more explicit formulation of strategies and explanations of these, or allowed children to explore them for themselves, in either case promoting a further shift to E3 level. This would correspond well with the switch in emphasis in adult input from prompts to explanations in Study 1. It would also explain why, in Study 1, 
where explicit understanding was initially somewhat higher, adults moved rapidly to provision of explanations, and children were able to appropriate these, whereas in Study 2 adult support was more basic.

This account implies that the RR model requires modification in various respects. First, rather than redescription being an exclusively endogenous process, exogenous counterparts need to be accorded a central role. There is little previous empirical work other than that of Pine and colleagues that has attempted to explore this role. Second, rather than children moving between distinct levels of representation, it would be more accurate to portray development as a shift along a continuum of explication and redescription, albeit one with some reasonably defined markers. One pointer in this direction is that the status of explanations as opposed to verbal encoding of strategies is left unclear in the current model, since both are treated as E3 level. The present research did not differentiate between these in terms of the justification index, but both types of response were apparent, and in terms of input, explanations appear to be more powerful (cf. Pine et al., 1999). This suggests that explanations should be identified with some form of E3+ level of representation, a true conceptual level, underpinning fully flexible usage. There is a bigger underlying issue here, concerning the nature of the shift from procedural to conceptual representation, when this can be said to have occurred, and how far there is continuity between the two. Whilst the present research did not address this directly, it may be noted that this issue is more apparent in real world problem-solving, where performance is not so much a matter of identifying a single correct strategy as choosing between alternative possibilities, and so having to consider rationale more explicitly.

It is important to conclude by considering the implications of the present research for pedestrian training more generally. The first point to note is that the findings reported here undoubtedly have broader applicability. Whilst the focus was on a single skill, they are wholly consistent with past research on the effects of training early primary age children in safe place location (Ampofo-Boateng et al., 1993; Thomson \& Whelan, 1997), and confirm two crucial points indicated by that work. The first is that the key process driving progress in pedestrian skill acquisition is the development of procedural strategies into conceptual (i.e. explanatory) representational frameworks, since it is these that underlie true generalisation and flexible deployment. This means 
that, provided this dimension is preserved, training need not necessarily take place at the roadside (though this will arguably provide the best basis for linguistic encoding and for refinement of skills). Thus, for instance, as the studies reported here indicate, computer-based training using simulations may be both effective and more efficient, as long as such materials are embedded within appropriate tasks.

The second point is that this implies a need to attend to methods carefully. Attempts to promote pedestrian skill that ignore the conceptual dimension, for example by simply modelling appropriate behaviour (Rivara, Booth, Bergman, Rogers \& Weiss, 1991) will fail to produce the same gains. Tasks which support the forms of social interaction that promote representational growth, on the other hand, will have a distinct advantage. In particular, where adults work with small groups, and both scaffolding and peer discussion are present, the rate of developmental change is likely to be substantially increased. The scale of the benefits observed here was admittedly relatively modest, but this is unsurprising given the exploratory nature of the research. What is significant is that the effects were systematic, and indicate how the impact of training of this kind might be increased, via use of materials which provide focused encouragement for productive interactions by adults trained in scaffolding and thus well-placed to capitalise on such resources. Again, computer-based materials may prove particularly apposite for this purpose, given the dynamic nature of the support they can afford. Armed with a clearer understanding of the learning processes involved, testing of hypotheses about ways of optimising training outcomes should be the next step for pedestrian skills research with children. 


\section{References}

Ampofo-Boateng, K. \& Thomson, J.A. (1990). Child pedestrian accidents: a case for preventative medicine. Health Education Research: Theory and Practice, 5, 265-274.

Ampofo-Boateng, K., Thomson, J.A., Grieve, R., Pitcairn, T.K., Lee, D.N. \& Demetre, J.D. (1993). A developmental and training study of children's ability to find safe routes to cross the road. British Journal of Developmental Psychology, 11, 31-45.

Bechtel, W. \& Abrahamsen, A. (1991). Connectionism and the Mind. Oxford: Blackwell.

Damon, W. \& Phelps, E. (1989). Critical distinctions among three approaches to peer education. International Journal of Educational Research, 13, 9-19.

Demetre, J. D., Lee, D.N., Grieve, R., Pitcairn, T.K., Ampofo-Boateng, K. \& Thomson, J.A. (1993). Young children's learning on road-crossing simulations. British Journal of Educational Psychology, 63, 348-358.

Doise, W. and Mugny, G. (1984). The Social Development of the Intellect. Oxford: Pergamon.

Gibson, J.J. (1966). The Senses Considered as Perceptual Systems. Boston: Houghton-Mifflin.

Howe, C.J. \& Tolmie, A. (1998). Productive interaction in the context of computersupported collaborative learning in science. In K. Littleton \& P. Light (Eds.), Learning with Computers: Analysing Productive Interaction. Pp.24-45. London: Routledge.

Howe, C.J., Tolmie, A., Duchak-Tanner, V. \& Rattray, C. (2000). Hypothesis testing in science: group consensus and the acquisition of conceptual and procedural knowledge. Learning \& Instruction, 10, 361-391. 
Howe, C.J., Tolmie, A., Greer, K. \& Mackenzie, M. (1995). Peer collaboration and conceptual growth in physics: task influences on children's understanding of heating and cooling. Cognition \& Instruction, 13, 483-503.

Howe, C.J., Tolmie, A., \& Rodgers, C. (1992). The acquisition of conceptual knowledge in science by primary school children: group interaction and the understanding of motion down an incline. British Journal of Developmental Psychology, 10, 113-130.

Karmiloff-Smith, A. (1992). Beyond Modularity: a Developmental Perspective on Cognitive Science. Cambridge, Mass.: MIT Press.

Kruger, A.C. (1993). Peer collaboration: conflict, co-operation or both? Social Development, 2, 165-182.

Mercer, N., Wegerif, R. \& Dawes, L. (2003). Using ICT to promote learning conversations. Nuffield Foundation Seminar, London.

Miell, D. \& MacDonald, R. (2000). Children's creative collaborations: the importance of friendship when working together on a musical composition. Social Development, 9, 348-369.

Piaget, J. (1932). The Moral Judgement of the Child. London: Routledge \& Kegan Paul.

Piaget, J. (1985). The equilibration of cognitive structures. Chicago: University of Chicago Press.

Pine, K.J. \& Messer, D.J. (1998). Group collaboration effects and the explicitness of children's knowledge. Cognitive Development, 13, 109-126.

Pine, K.J. \& Messer, D.J. (1999). What children do and what children know: looking beyond success using Karmiloff-Smith's RR framework. New Ideas in Psychology, 17, 17-30. 
Pine, K.J., Messer, D.J. \& Godfrey, K. (1999). The teachability of children with naïve theories: an exploration of the effects of two teaching methods. British Journal of Educational Psychology, 69, 201-211.

Rivara, F.P., Booth,C.L., Bergman, A.B., Rogers, L.W. \& Weiss, J. (1991).

Prevention of pedestrian injuries to children: effectiveness of a school training program. Pediatrics, 88, 770-775.

Rothengatter, T. (1984). A behavioural approach to improving traffic behaviour of young children. Ergonomics, 2, 147-160.

Ryhammer, L. \& Bergland, G.W. (1980). Children and instruction in road safety. Uppsala Reports on Education, No. 8. University of Uppsala, Sweden.

Thomson, J.A., Tolmie, A., Foot, H.C. \& McLaren, B. (1996). Child development and the aims of road safety education: a review and analysis. Department of Transport Road Safety Research Report No. 1. London: HMSO.

Thomson, J.A. \& Whelan, K.M. (1997). A Community Approach to Road Safety Education Using Practical Training Methods: The Drumchapel Report. Road Safety Research Report No. 2. London: H.M.S.O.

Tolmie, A. \& Howe, C.J. (1993). Gender and dialogue in secondary school physics. Gender \& Education, 5, 191-209.

Tolmie, A. \& Howe, C.J. (1994). Computer-directed group activity and the development of children's hypothesis-testing skills. In H.C. Foot, C.J. Howe, A. Anderson, A. Tolmie \& D. Warden (Eds.) Group and Interactive Learning. pp.139144. Southampton: Computational Mechanics Publications.

Tolmie, A., Howe, C.J., Duchak-Tanner, V. \& Rattray, C. (1999). Explicit and implicit support for procedural learning within collaborative group work in science. Ixth European Conference on Developmental Psychology, Spetses, Greece. 
Tolmie, A., Howe, C.J., Mackenzie, M. \& Greer, K. (1993). Task design as an influence on dialogue and learning: primary school group work with object flotation. Social Development, 2, 183-201.

Tolmie, A.K., Thomson, J.A., Foot, H.C., McLaren, B. \& Whelan, K.M. (1998). Problems of attention and visual search in the context of child pedestrian behaviour. Department of the Environment, Transport and the Regions Road Safety Research Report No. 8. London: DETR.

Tolmie, A.K., Thomson, J.A., Foot, H.C., Whelan, K.M., Sarvary, P. \& Morrison, S. (2002). Development and Evaluation of a Computer-Based Pedestrian Training Resource for Children Aged 5 to 11 Years. Department for Transport, Local Government and the Regions Road Safety Research Report No. 27. London: DTLR.

Tomasello, M. (1999). The Cultural Origins of Human Cognition. Cambridge, MA: Harvard University Press.

Tomasello, M., Kruger, A. \& Ratner, H. (1993). Cultural learning. Behavioral and Brain Sciences, 16, 495-552.

Vygotsky, L.S. (1978). Mind in Society: The Development of Higher Psychological Processes. Cambridge, MA: Harvard University Press.

Webb, N. (1989). Peer interaction and learning in small groups. International Journal of Educational Research, 13, 21-39.

Williams, J.M. \& Tolmie, A. (2000). Conceptual change in biology: group interaction and the understanding of inheritance. British Journal of Developmental Psychology, 18, 625-649.

Wood, D. (1986). Aspects of teaching and learning. In M. Richards \& P. Light (Eds.), Children of Social Worlds: Development in a Social Context. Cambridge: Polity. 
Table 1. Pre-test scores and pre- to post-test change by intervention condition (Study 1) on the computer report, roadside report and roadside crossing decision tasks (standard deviations in parentheses; values within change indices with no common subscript differ significantly).

$\mathrm{N}^{1}$

\section{Computer report task:}

R

I

Ratio*

Justifications

\section{Roadside report task:}

$\mathrm{R}$

I

Ratio*

Justifications

\begin{tabular}{cccccc} 
Adult Guidance & \multicolumn{2}{c}{ Peer Discussion } & \multicolumn{2}{c}{ Control } \\
Pre-test & Change & Pre-test & Change & Pre-test & Change \\
$(18)$ & $(17)$ & $(17)$ & $(17)$ & $(14)$ & $\left(13 / 12^{+}\right)$
\end{tabular}

(17)

(17)

(17)

(14) $\quad\left(13 / 12^{+}\right)$ 
Table 2. Dialogue frequencies and performance scores across training sessions in the peer discussion and adult guidance conditions (Study 1).

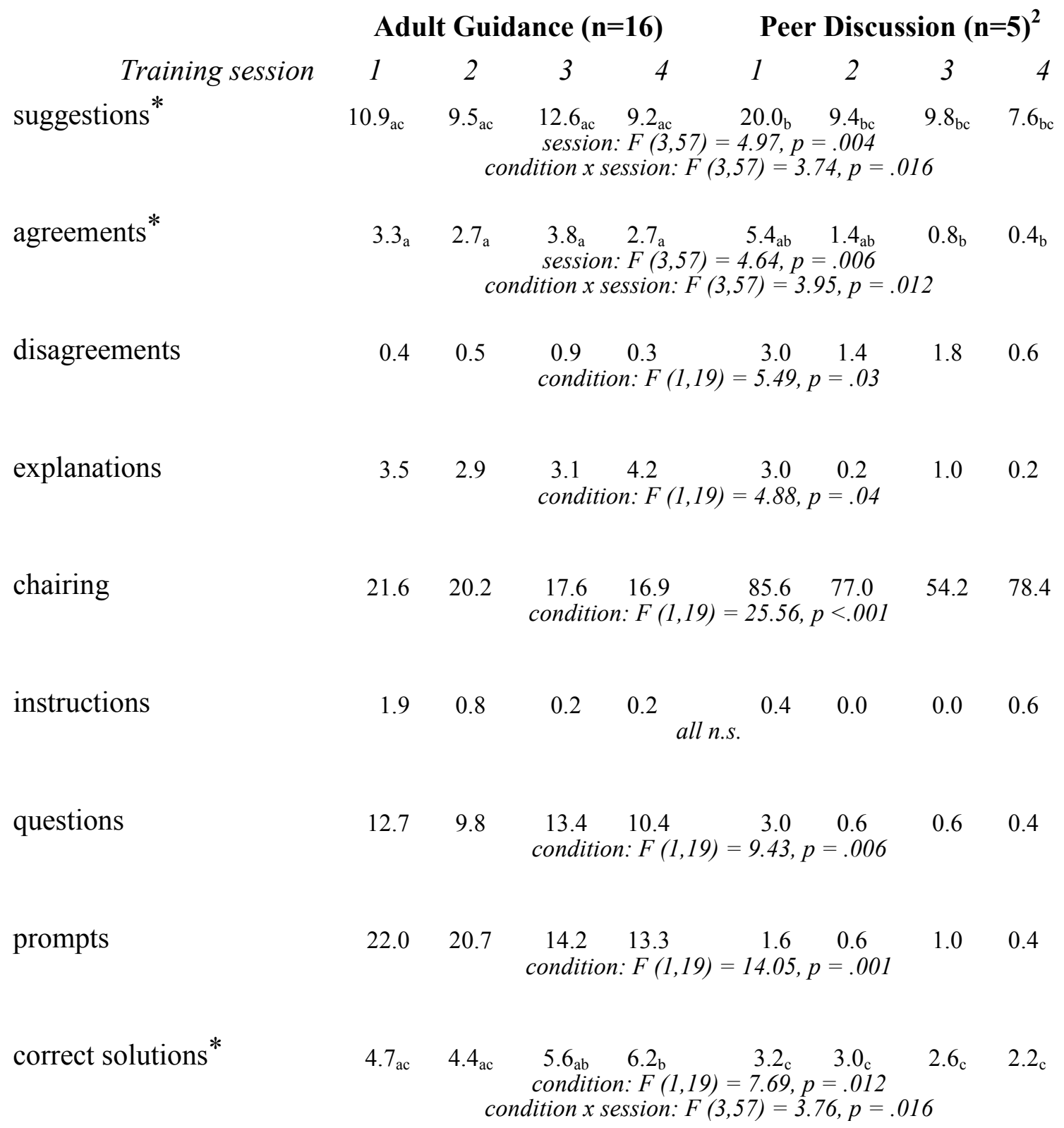

* for significant interactions, where no subscript is shared, values are significantly different at $\mathrm{p}$ $<.005$ on one-tailed t-test with Bonferroni correction

\footnotetext{
${ }^{2}$ Due to camera and microphone faults, records of three sessions, two adult guidance and one peer discussion, were lost. Since data for these sessions was missing, the relevant dyads/groups had to be excluded from both the session by session and overall dialogue analyses; the means presented here are based only on those dyads/groups that were included in these analyses. The combined effects of missing data at pre- and post-test, and during training meant that analyses of the effects of interaction on learning were based on 30 children, 16 from the adult guidance condition, and 14 from the peer discussion.
} 
Table 3. Incidence of explanations across training sessions in the adult guidance condition (Study 1).

i. Percentage of explanations provided by adults and by children (mean percentages in parentheses)

$\begin{array}{lcccc}\text { Adults } & 78 \%(72 \%) & 65 \%(64 \%) & 65 \%(44 \%) & 55 \%(55 \%) \\ \text { Children } & 22 \%(22 \%) & 35 \%(30 \%) & 35 \%(25 \%) & 45 \%(45 \%)\end{array}$

ii. Mean percentage of adult explanations occurring after each of four categories of dialogue

$\begin{array}{cccc}\text { Session } 1 & \text { Session } 2 & \text { Session 3 } & \text { Session 4 } \\ 16 \% & 15 \% & 6 \% & 8 \% \\ 14 \% & 21 \% & 0 \% & 16 \% \\ 27 \% & 11 \% & 28 \% & 26 \% \\ 0 \% & 21 \% & 18 \% & 8 \%\end{array}$

iii. Mean percentage of child explanations occurring after each of four categories of dialogue

$\begin{array}{cccc}\text { Session } 1 & \text { Session } 2 & \text { Session } 3 & \text { Session } 4 \\ 5 \% & 14 \% & 22 \% & 12 \% \\ 25 \% & 32 \% & 18 \% & 40 \% \\ 0 \% & 6 \% & 4 \% & 10 \% \\ 0 \% & 0 \% & 0 \% & 8 \%\end{array}$


Table 4. Pre-test scores and pre- to post-test change by intervention condition (Study 2) on the computer report task (standard deviations in parentheses).

\begin{tabular}{|c|c|c|c|c|c|c|}
\hline & \multicolumn{2}{|c|}{ Adult-child } & \multicolumn{2}{|c|}{ Adult-group } & \multicolumn{2}{|c|}{ Control } \\
\hline $\mathrm{N}$ & $\begin{array}{l}\text { Pre-test } \\
\text { (24) }\end{array}$ & $\begin{array}{c}\text { Change } \\
\text { (24) }\end{array}$ & $\begin{array}{c}\text { Pre-test } \\
\text { (24) }\end{array}$ & $\begin{array}{c}\text { Change } \\
\text { (24) }\end{array}$ & $\begin{array}{l}\text { Pre-test } \\
\text { (15) }\end{array}$ & $\begin{array}{c}\text { Change } \\
\text { (15) }\end{array}$ \\
\hline \multirow[t]{2}{*}{$\mathrm{R}$} & 29.77 & +5.19 & 31.17 & +6.27 & 34.40 & +1.27 \\
\hline & (10.81) & (10.90) & (11.14) & (13.88) & $(9.63)$ & (8.98) \\
\hline \multirow[t]{2}{*}{ I } & 37.15 & -7.69 & 39.04 & -9.81 & 46.67 & +2.33 \\
\hline & (15.87) & (16.36) & (14.67) & (18.89) & (19.54) & (20.90) \\
\hline \multirow[t]{2}{*}{ Ratio } & -.09 & +.22 & -.10 & +.30 & -.12 & .00 \\
\hline & $(0.25)$ & $(0.31)$ & $(0.28)$ & (.35) & $(0.15)$ & $(0.31)$ \\
\hline \multirow[t]{2}{*}{ Justifications } & 0.08 & +0.62 & 0.08 & +0.71 & 0.07 & +0.40 \\
\hline & $(0.41)$ & $(1.86)$ & $(0.41)$ & $(1.40)$ & $(0.26)$ & (1.30) \\
\hline
\end{tabular}


Table 5. Dialogue frequencies and performance scores across training sessions in the adult-child and adult-group conditions (Study 2) [NB all effects ns unless stated].

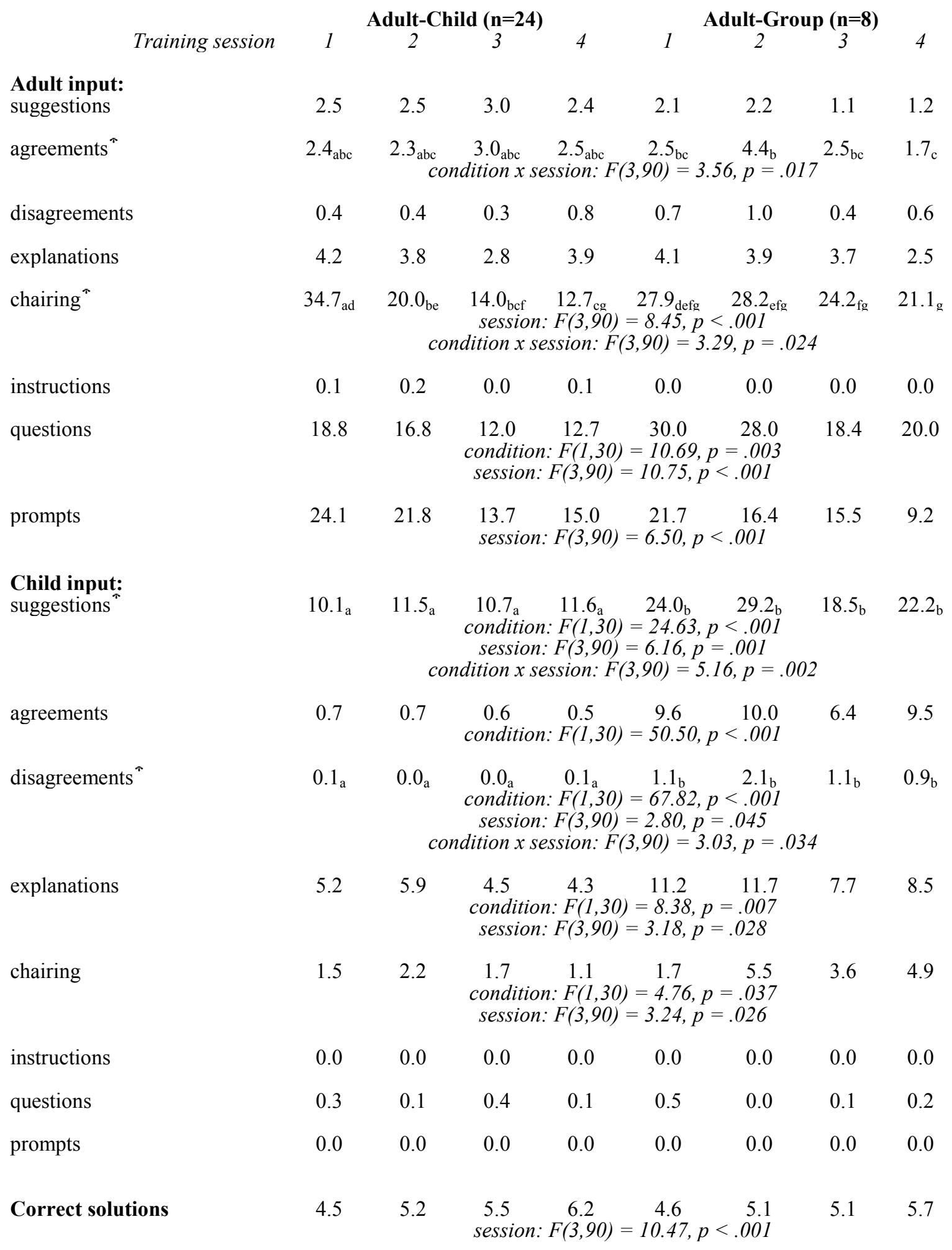

* for significant interactions, where no subscript is shared, values are significantly different at $\mathrm{p}$ $<.005$ on one-tailed t-test with Bonferroni correction 